\title{
Game theoretic approach for joint transmit beamforming and power control in cognitive radio MIMO broadcast channels
}

\author{
Ju Ni ${ }^{1}$ and Hailin $\mathrm{XiaO}^{2,3^{*}}$
}

\begin{abstract}
In this paper, we present a game-theoretic approach to the problem of joint transmit beamforming and power control in cognitive radio (CR) multiple-input multiple-output broadcast channels (MIMO-BCs), where the primary users (PUs) coexist with the secondary users (SUs) and share the same spectrum. The cognitive base station (CBS) is equipped with multiantenna and transmits independent data streams to several decentralized single-antenna terminals. Our design goal is to jointly adjust the beamformers and transmission powers according to individual SINR (signal-to-interference-plus-noise ratio) requirements in order to meet SINR balancing for CR MIMO-BCs. In this context, two problems need to be solved: (1) the design beamforming must enable a balancing of the SINR among all SUs for a fixed total power of CBS and (2) the total transmission power must be minimized while satisfying a set of SINR constraints for fixed beamformers. The proposed approach is an application of separable games, where beamforming vectors are modeled as beamforming subgame and power control is modeled as power control subgame. We then use the convex theory of noncooperative game to solve the optimalization problem. Finally, we propose an iterative algorithm to reach Nash equilibrium (NE) of the joint beamforming subgame and power control subgame. Numerical results are provided to validate the optimality and the convergence of the proposed algorithm.
\end{abstract}

Keywords: Multiple-input multiple-output (MIMO), Cognitive radio network, Beamforming, Power allocation, Game theory

\section{Introduction}

Cognitive radio networks (CR-Nets) have received tremendous attention due to its capability of compensating for the spectrum scarcity. The original idea of $\mathrm{CR}$ allows secondary users (SUs) to access the spectrum that is originally allocated to primary users (PUs) when the spectrum is not in use [1-4]. To support this functionality, SUs are required to precisely sense the radio environment and detect the presence of $\mathrm{PU}$, which is proved not to be an easy task $[5,6]$. Alternatively, the idea of spectrum sharing in CR-Nets $[7,8]$ allows simultaneous transmissions of PUs and SUs, provided that the quality-of-service (QoS) of PUs is guaranteed. Recently, the multiple antenna

\footnotetext{
*Correspondence: xhl xiaohailin@163.com

${ }^{2}$ Guangxi Key Laboratory of Wireless Wideband Communication and Signal Processing, Jinji Road, 541004 Guilin, China

${ }^{3}$ National Mobile Communications Research Laboratory, Southeast University, 210096 Najing, China

Full list of author information is available at the end of the article
}

technique is introduced to CR-Nets $[9,10]$. By employing multiple antennas, the concurrent spectrum sharing can be performed without any degradation in QoS for PUs [11].

In CR multiple-input multiple-output broadcast channels (CR MIMO-BCs), the cognitive base station (CBS) is equipped with multiple antennas and sends data to multiple SUs simultaneously in the same system resource, where multiple antennas are employed at the transmitter to facilitate spatial multiplexing. Appropriately designing transmit beamforming for various spatial multiplexing designs have been investigated to steer energy in the directions of the intended users [12-15]. In [12], the equivalent transmit beamforming matrix has been designed to minimize the effect of the resulted interference on the PU transmissions. However, this approach

\section{Springer Open}

(c) $2016 \mathrm{Ni}$ and Xiao. Open Access This article is distributed under the terms of the Creative Commons Attribution 4.0 International License (http://creativecommons.org/licenses/by/4.0/), which permits unrestricted use, distribution, and reproduction in any medium, provided you give appropriate credit to the original author(s) and the source, provide a link to the Creative Commons license, and indicate if changes were made. 
is not robust enough as there are some uncertainties in both the CR transmit covariance matrix and the degrees of freedom of CR MIMO system. In [13, 14], robust downlink beamforming for multiuser CR-MISO system has been investigated. However, it is quite difficult to predict in advance whether the problem with given SINR (signal-to-interference-plus-noise ratio) targets, interference thresholds, and total power budget is feasible. To avoid infeasibility issues, some beamforming techniques have been developed, for example, game theory that has been proven to be successful in beamforming design [15], as will be investigated in this paper.

To guarantee the QoS of PUs in the CR-Nets, both the total power constraint and the individual interference power constraint applied at each primary receiver are considered. Joint beamforming and power control have been accepted as an efficient interference suppression technique in CR-Nets $[16,17]$. In this paper, based on joint beamforming and power control, we focus on the SINR balancing problem [18] in CR MIMO-BCs. This problem is of particular interest since the following issues affect the SINRs of SUs.

First, transmit beamforming at CBS is more complicated in that the beamformers must be optimized jointly. This is because SUs' beamformers cannot be separately designed, as the interference introduced to each SU's beamformer is a function of all the beamformer vectors and power allocation. We tackle this problem by introducing separable game [19] to the design framework, as explained in Section 3. Partially related results have been found in [20-22]. In [20,21], under the sumpower constraint, an alternating optimization strategy for joint power control or linear beamforming has been proposed to solve the SINR balancing problem. Although this beamforming strategy is optimal, identifying an eigenvalue optimization problem for an extended coupling matrix poses heavy computational burden. In [22], subject to interference constraints of PUs and peak transmission power constraints of SUs, a jointing beamforming and power control algorithm called decoupled multipleconstraint power allocation has been presented to balance the SINR level for all SUs. However, when large numbers of active PUs and SUs exist, the computation complexity is still quite high. Our first contribution of this paper is to solve SINR balancing problem in CR MIMO-BCs with much lower complexity.

Second, due to the coupled structure of the transmitted signals, the $\mathrm{BC}$ optimization problems are usually non-convex and thus cannot be solved directly. To tackle this difficulty, the non-convex $\mathrm{BC}$ problem is transformed into a convex multiple-access channel (MAC) problem via a so-called BC-MAC duality relationship [23]. Under a single sum-power constraint or a set of linear power constraint, the problem of $\mathrm{BC}$ can be solved as a minimax optimization problem in its dual MAC setting [24]. Using a minimax optimization approach, the sum-capacity for the MIMO-BCs has been studied in [25, 26], which seeks to maximize the minimal SNR among all the users under the sum-power constraint by transforming into its dual MAC problem. In this paper, the minimax optimization problem is also investigated. We show that the proposed method can handle multiple linear power constraint of CR MIMO-BCs and is essentially equivalent to the method based on SINR balancing.

The remainder of this paper is organized as follows. Section 2 describes the system model and formulates the problem. Section 3 forms joint transmit beamforming and power control as noncooperative separable game. Section 4 proposes an iterative algorithm that achieves the optimal Nash equilibrium (NE) of joint transmit beamforming subgame and power control subgame. Section 5 provides numerical results and the related discussions. Conclusions are drawn in Section 6.

Notation: Vectors and matrices are denoted by lowercase and uppercase boldface letters, respectively. $(\cdot)^{T}$, $(\cdot)^{H}$, and $\operatorname{tr}(\cdot)$ denote transpose, conjugate transpose, and trace of a matrix. $\|\cdot\|_{2}$ represents the Euclidean norm of a vector, and $E[\cdot]$ is the expectation. $\mathbf{I}_{M}$ is an $M \times M$ identity matrix, and $\{\cdot\}$ denotes the subset.

\section{System model and problem formulation}

\subsection{System model}

The downlink scenario of a single-cell CR-Net coexisting with a single-cell PR-Net is depicted in Fig. 1, in which the system model of CR MIMO-BCs is shown in detail in Fig. 2, where the cognitive base station (CBS) is equipped with $M$ antennas and transmits independent data to $K$ different SUs, each with one antenna. For simplicity, we also assume that the primary base station (PBS) and $N$ PUs are equipped with a single antenna. Block diagram of $\mathrm{BC}-\mathrm{MAC}$ duality relationship for the transmission process is shown in Fig. 3. In Fig. 1, the CR-Net is installed far enough from PBS, which guarantees that the amount of interference power to each PU receiver is kept below a certain threshold [7]. Although PBS is interfering with the normal operations of CR-Net, the power received by SUs from CBS is much larger than the power received from PBS, i.e., the interfering power from PBS can be accumulated as a part of its noise term [13]. Hence, the received signal at the $k$ th $\mathrm{SU}\left(\mathrm{SU}_{k}\right)$ is determined by

$$
y_{k}=\mathbf{h}_{k}^{H} \mathbf{x}+n_{k}
$$

for $k=1, \cdots, K$, where $\mathbf{h}_{k} \in\left\{\mathbb{C}^{M \times 1}\right\}_{k=1}^{K}$ represents the channel from CBS to $\mathrm{SU}_{k}$, and $\left\{n_{k}\right\}_{k=1}^{K}$ are white Gaussian 




Fig. 1 The downlink scenario of a single-cell CR-Net coexisting with a single-cell PR-Net

noise terms with $n_{k} \sim C N\left(0, \sigma_{k}^{2}\right)$. The signal transmitted by the CBS is given by

$$
\mathbf{x}=\sum_{k=1}^{K} \sqrt{p_{k}} \mathbf{w}_{k} s_{k}=\tilde{\mathbf{U}} \mathbf{s}
$$

where $\tilde{\mathbf{U}}=\left[\sqrt{p_{1}} \mathbf{w}_{1}, \sqrt{p_{2}} \mathbf{w}_{2}, \cdots, \sqrt{p_{k}} \mathbf{w}_{k}\right]$ and $\mathbf{s}=$ $\left[s_{1}, s_{2}, \cdots, s_{k}\right]^{T}$ is the independent data streams. We also assume that the data streams for different SUs are independent of each other and $E\left[\left|s_{k}\right|^{2}\right]=1$. Using $\left\{\mathbf{w}_{k} \in \mathbb{C}^{M \times 1}\right\}_{k=1}^{K}$ as beamforming vectors for $\mathrm{SU}_{\mathrm{k}}, \mathbf{w}_{k}$ satisfies $\left\|\mathbf{w}_{k}\right\|_{2}=1$. Moreover, $p_{k}$ is the power allocation to $\mathrm{SU}_{k}$ and is subject to a peak power constraint $\widehat{p}_{k}$, i.e., $p_{k} \leq \widehat{p}_{k}$.

In this paper, it is assumed that CBS has a perfect channel state information (CSI). Here, the protocol of CBS can be designed as follows: each frame contains sensing

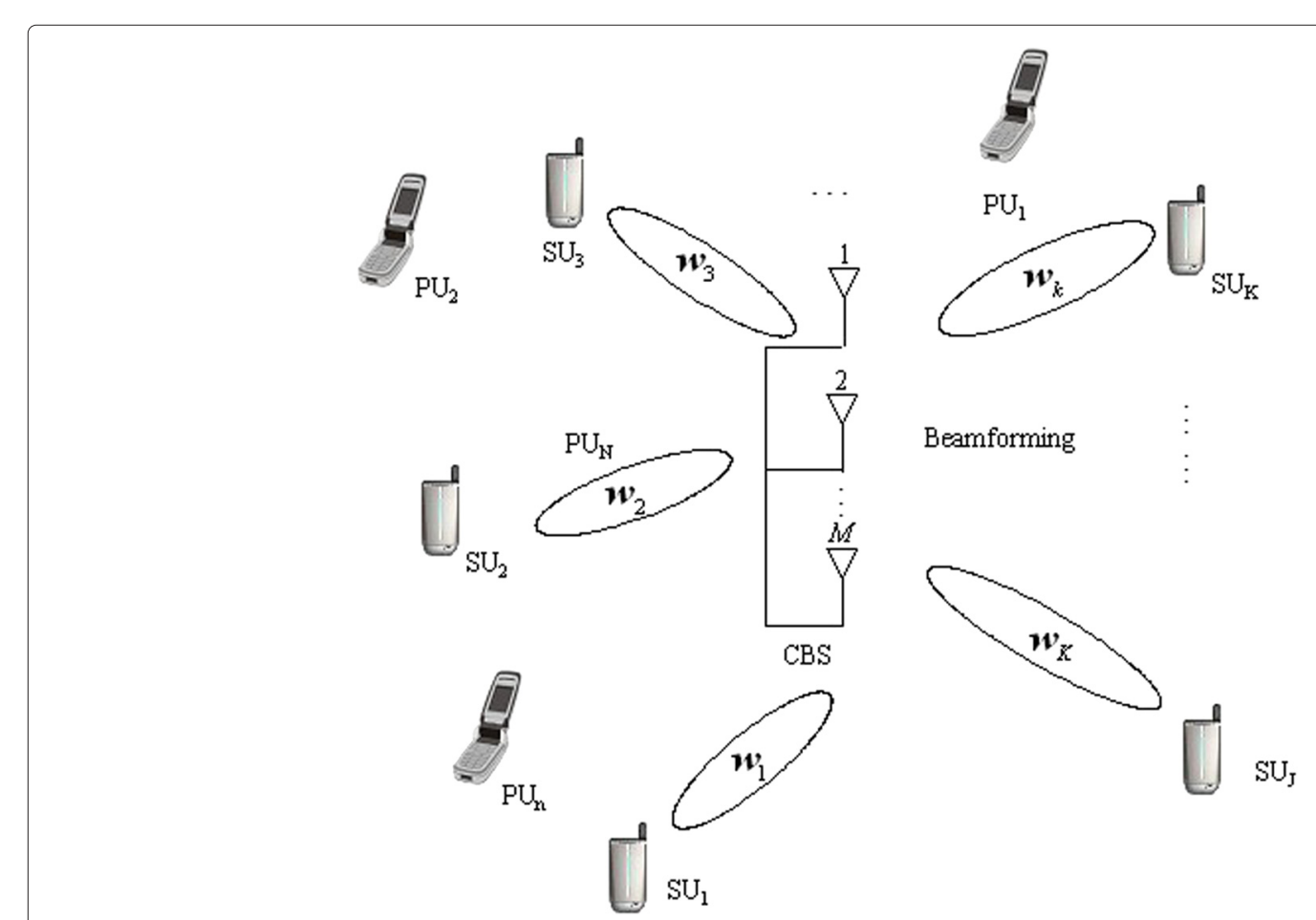

Fig. 2 The system model of CR MIMO-BCS 


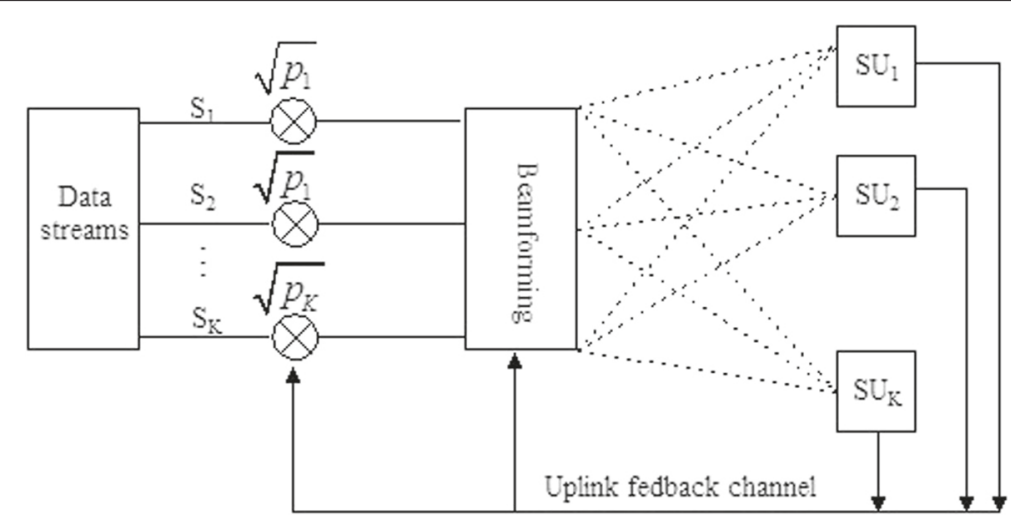

Fig. 3 Block diagram of BC-MAC duality relationship for the transmission process

sub-frame and data transmission sub-frame. During the sensing sub-frame, SUs remain silent, and thus CBS can detect spectrum and measure the effect from PUs and noise. During the first portion of the data transmission sub-frame, CBS transmits training sequences to SUs so that CBS can estimate downlink CSI. Although we confine ourselves to a single-cell system in which all beamformers belong to a CBS, this model can easily be extended so as to incorporate multiple CBS. Let $g_{n, k}$ be the power gain between CBS and the $n$th $\mathrm{PU}\left(\mathrm{PU}_{n}\right)$, the interference power received by $\mathrm{PU}_{n}$ is

$$
I_{n}=\sum_{k} g_{n, k} p_{k} \mathbf{w}_{k}^{H} \mathbf{w}_{k}
$$

To ensure the QoS of PUs, the interference power should be below certain thresholds. Let $p_{\text {th }}^{(n)}$ represents the interference power threshold for $\mathrm{PU}_{\mathrm{n}}$, i.e., $I_{n} \leq p_{\mathrm{th}}^{(n)}$. Substituting (2) into (1), the received signal of $\mathrm{SU}_{k}$ is expressed as

$$
y_{k}=\sqrt{p_{k}} \mathbf{h}_{k}^{H} \mathbf{w}_{k} s_{k}+\sum_{i \neq k} \sqrt{p_{i}} \mathbf{h}_{k}^{H} \mathbf{w}_{i} s_{i}+n_{k}
$$

where the first term is the received signal of the intended message, whereas the second and third terms denote the interference from other messages and noise, respectively. The SINR at $\mathrm{SU}_{k}$ is given by $[9,27]$

$$
\operatorname{SINR}_{k}\left(\mathbf{w}_{k}, \mathbf{p}\right)=\frac{p_{k} \mathbf{w}_{k}^{H} \mathbf{R}_{k} \mathbf{w}_{k}}{\sum_{i \neq k} p_{i} \mathbf{w}_{i}^{H} \mathbf{R}_{k} \mathbf{w}_{i}+\sigma_{k}^{2}}, \text { subject to } I_{n} \leq p_{\mathrm{th}}^{(n)},
$$

where $\mathbf{R}_{k}=\mathbf{h}_{k} \mathbf{h}_{k}^{H}$ and $\sum_{i \neq k} p_{i} \mathbf{w}_{i}^{H} \mathbf{R}_{k} \mathbf{w}_{i}$ is interference of all the SUs with the exception the $k$ th SU.

\subsection{Problem formulation}

Uplink-downlink duality is identified as SINR transformation between BC-MAC [26]. Based on this duality, maximizing the minimum user SINR in the uplink can be done straightforwardly since the beamformers can be optimized individually and SINRs are only coupled by the user's transmit power [28]. In contrast, downlink optimization is generally a nontrivial task because the user SINRs depend on all optimization variables and have to be optimized jointly. In this work, we propose to solve the problem of transmit beamforming and power control jointly by simultaneously maximizing the total numbers of SUs that can be served in the same slot at their QoS and minimizing the total power required to serve them. However, to maintain user fairness, their SINRs should be balanced and maximized.

Motivated by above consideration, we formulate the SINR balancing [29] designs of CR MIMO-BCs into minimax optimization problems, where each SINR balancing depends on the choice of all beamformers and transmission powers. Thus, a necessary and sufficient condition for global achievability is obtained by maximizing $\min _{k} \mathrm{SINR}_{k} / \gamma_{k}$ over all possible beamformers and transmission powers. Therefore, the SINR balancing problem for CR MIMO-BCs is defined as

$$
\begin{aligned}
& \max _{\mathbf{w}_{k}, \mathbf{p}} \min _{1 \leq k \leq K} \frac{\operatorname{SINR}_{k}\left(\mathbf{w}_{k}, \mathbf{p}\right)}{\gamma_{k}} \\
& \text { subject to } 0 \leq p_{k} \leq \widehat{p}_{k},\|\mathbf{p}\|_{1} \leq p \\
& I_{n} \leq p_{\text {th }}^{(n)}
\end{aligned}
$$

where $\gamma_{k}$ is the target SINR for $\mathrm{SU}_{k}$. The object function (6) is to find power allocation such that all SUs can achieve their target SINRs in a fair manner.

\section{Joint transmit beamforming and power control as noncooperative separable game}

In this section, we form joint transmit beamforming and power control as noncooperative separable game, where beamforming vectors are modeled as beamforming subgame and power control is modeled as power control subgame. We target at jointly adjusting the beamformers and transmission powers according to individual SINR 
requirements in order to meet SINR balancing for $C R$ MIMO-BCs.

\subsection{Noncooperative beamforming game}

The beamforming design techniques based on the satisfaction of target SINRs have been investigated in [18], where the approaches use the criterion of minimizing the total power of beamformer subject to SINR constraints for SUs and interference leakage constraints for PUs. However, the resulting problems can be infeasible. In such cases, to make the problem feasible, the design has to be repeated with lower SINR targets or some SUs have to be dropped [12, 27]. A more attractive problem formulation which always guarantees feasibility is based on SINR balancing with extra PU interference leakage constraints. In this paper, to make the problem feasible, the beamforming design adopts target SINR $\gamma_{k}$ for $\mathrm{SU}_{k}$. A set of optimal beamforming vectors exists for feasible $\gamma_{k}$, and the optimization problem is written as

$$
\underset{\tilde{\mathbf{w}}_{k}}{\operatorname{Minimize}} \sum_{k=1}^{K}\left\|\tilde{\mathbf{w}}_{k}\right\|^{2}
$$

where $\tilde{\mathbf{w}}_{k}=\sqrt{p_{k}} \mathbf{w}_{k}$. The SINR-based beamforming designed for downlink transmission immediately carry over to uplink reception [18]. Using the principle of uplink-downlink duality, the beamformer designed in the virtual uplink mode can be used in the downlink mode to achieve the same SINR values by choosing appropriate downlink power allocations. Thus, the solution of the downlink beamforming problem (9) is equivalently obtained by solving an easier-to-handle uplink problem instead. The game theory is usually suitable to solve beamforming problem where distributed decision making is required [30]. According to uplink-downlink duality, we also adopt the separable game theory to solve beamforming problem.

The general structure of the optimal beamforming is obtained via a network Lagrangian, which is commonly referred to as the minimum variance distortionless response (MVDR) beamforming [31,32]. Now, we define normalized MVDR beamforming vector at $\mathrm{SU}_{k}$ by

$$
\mathbf{w}_{k}^{\dagger}=\mathbf{h}_{k}^{H} \mathbf{R}_{k}\left(\tilde{\mathbf{w}}_{-k}\right)^{-1} \mathbf{h}_{k} \mathbf{w}_{k}
$$

where $\tilde{\mathbf{w}}_{-k}=\left\{\tilde{\mathbf{w}}_{k}:-k \triangleq 1, \cdots, k-1, k+1, \cdots, K\right\}$. The interferer plus noise covariance at $\mathrm{SU}_{k}$ is given by

$$
\mathbf{R}_{k}\left(\tilde{\mathbf{w}}_{-k}\right)=\sum_{i \neq k, i=1}^{K} \mathbf{h}_{k} \tilde{\mathbf{w}}_{i}^{H} \tilde{\mathbf{w}}_{i} \mathbf{h}_{k}^{H}+\sigma^{2} \mathbf{I} .
$$

Substituting (10) into (5) and imposing the constraints, the corresponding Lagrangian is obtained as

$$
\begin{gathered}
L(\mathbf{w})=\sum_{k=1}^{K}\left\|\tilde{\mathbf{w}}_{k}\right\|^{2}+\sum_{k=1}^{K} \tau_{k}\left(\gamma_{k}-\tilde{\mathbf{w}}_{k}^{H} \mathbf{h}_{k}^{H} \mathbf{R}_{k}^{-1} \mathbf{h}_{k} \tilde{\mathbf{w}}_{k}\right) \\
\sum_{k=1}^{K} \tau_{k}\left(\gamma_{k}-\tilde{\mathbf{w}}_{k}^{H} \mathbf{h}_{k}^{H} \mathbf{R}_{k}^{-1} \mathbf{h}_{k} \tilde{\mathbf{w}}_{k}\right)=0
\end{gathered}
$$

where $\tau_{k}$ is the Lagrangian parameter. We can obtain the optimal beamforming vector $\mathbf{w}_{k}$ by taking the derivative of $L(\mathbf{w})$ in (12) with respect to $\tilde{\mathbf{w}}_{k}$, we obtain

$$
\begin{aligned}
\frac{\partial L(\mathbf{w})}{\partial \tilde{\mathbf{w}}_{k}}= & 2 \tilde{\mathbf{w}}_{k}-2 \tau_{k} \mathbf{h}_{k}^{H} \mathbf{R}_{k}^{-1} \mathbf{h}_{k} \tilde{\mathbf{w}}_{k} \\
& +\sum_{i \neq k, i=1}^{K} \tau_{i} \tilde{\mathbf{w}}_{i} \mathbf{h}_{i}^{H} \mathbf{h}_{i} \mathbf{R}_{i}^{-1} \frac{\partial \mathbf{R}_{i}}{\partial \tilde{\mathbf{w}}_{k}} \mathbf{R}_{i}^{-1} \mathbf{h}_{i} \tilde{\mathbf{w}}_{i}=0
\end{aligned}
$$

where the identity $\partial \mathbf{R}_{i}^{-1} / \partial \tilde{\mathbf{w}}_{k}=-\mathbf{R}_{i}^{-1} \partial \mathbf{R}_{i} / \partial \tilde{\mathbf{w}}_{k} \mathbf{R}_{i}^{-1}$ is used. We simplify (14) and obtain

$\tilde{\mathbf{w}}_{k}-\tau_{k} \mathbf{h}_{k}^{H} \mathbf{R}_{k}^{-1} \mathbf{h}_{k} \tilde{\mathbf{w}}_{k}+\sum_{i \neq k, i=1}^{K} \tau_{i} \mathbf{h}_{i}^{H} \mathbf{R}_{i}^{-1} \mathbf{h}_{i} \tilde{\mathbf{w}}_{i} \tilde{\mathbf{w}}_{i}^{H} \mathbf{h}_{i}^{H} \mathbf{R}_{i}^{-1} \mathbf{h}_{i} \tilde{\mathbf{w}}_{k}=0$.

Substituting (10) into (15), the corresponding stationary points of the Lagrangian (13) are given by

$$
\mathbf{w}_{k}=\tau_{k} \mathbf{Q}_{k}^{-1} \mathbf{h}_{k}^{H} \mathbf{R}_{k}\left(\tilde{\mathbf{w}}_{-k}\right)^{-1} \mathbf{h}_{k} \mathbf{w}_{k}
$$

where matrix $\mathbf{Q}_{k}$ is defined as

$$
\mathbf{Q}_{k}=\sum_{k \neq i, i=1}^{K} \tau_{i} p_{i}^{*} \mathbf{h}_{i}\left(\mathbf{w}_{i}^{\dagger}\right)^{H} \mathbf{w}_{i}^{\dagger} \mathbf{h}_{i}^{H}+\sigma^{2} \mathbf{I} .
$$

Substituting (15) into (13), $\tau_{k}$ is given by

$$
\tau_{k}=\frac{\mathbf{w}_{k}^{H} \mathbf{Q}_{k} \mathbf{w}_{k}}{\mathbf{w}_{k}^{H} \mathbf{h}_{i}^{H} \mathbf{R}_{i}^{-1} \mathbf{h}_{i} \mathbf{w}_{k}} .
$$

As described above, we aim to solve the SINR balancing problem under the total transmission power constraint. We propose the first-level game that it is defined as

$$
\mathbf{w}_{k}=\arg \max _{\mathbf{w}} \mathbf{h}_{k}^{H} \mathbf{R}_{k}\left(\tilde{\mathbf{w}}_{-k}\right)^{-1} \mathbf{h}_{k} \mathbf{w}_{k} .
$$

Applying MMSE criterion, (19) can be rewritten as

$$
\mathbf{w}_{k}=\arg \max _{\mathbf{w}_{k}} \frac{\mathbf{h}_{k}^{H} \mathbf{R}_{k}\left(\tilde{\mathbf{w}}_{-k}\right)^{-1} \mathbf{h}_{k} \mathbf{w}_{k}}{\mathbf{w}_{k}^{H} \mathbf{R}_{k}^{*}\left(\tilde{\mathbf{w}}_{-k}\right)^{-1} \mathbf{w}_{k}} .
$$

With regard to (20), there is nonunique solution. Hence, it can be used to improve the result in an iterative 
algorithm to find the optimal beamforming vectors. We set $\mathbf{w}_{k}(m+1)=\left(\mathbf{w}_{k}^{\dagger}(m+1)\right)^{*}$ and obtain

$$
\mathbf{w}_{k}(m+1)=\mathbf{R}_{k}^{*}\left(\tilde{\mathbf{w}}_{-k}\right)^{-1} \mathbf{h}_{k}^{H} \mathbf{R}_{k}\left(\tilde{\mathbf{w}}_{-k}\right)^{-1} \mathbf{h}_{k} \mathbf{w}_{k}(m)
$$

where $m$ denotes iteration steps.

\subsection{The noncooperative power control game}

In this noncooperative game, the SU's beamforming vectors are fixed, and individual SUs adjust only their power in their corresponding strategy spaces in order to minimize the total transmission while satisfying a set of target SINR. The noncooperative beamforming subgame is modeled as:

1. Players: $\Gamma=\{1,2, \cdots, K\}$, where $k=1,2, \cdots, K$ represents $\mathrm{SU}_{k}$.

2. Action space: $\Omega=p_{1} \times p_{2} \cdots \times p_{K}$, where $p_{k}=\left[0, \widehat{p}_{k}\right]$ represents $\mathrm{SU}_{k}$ 's action set. The action vector of all SUs is denoted by $\mathbf{p}=\left[p_{1}, \cdots, p_{K}\right]$, where $p_{k} \in \Omega$. The action vector excluding the action of $\mathrm{SU}_{k}$ is customarily denoted by $\mathbf{p}_{-k}$.

3. Utility function: we use $u_{k}\left(p_{k}, \mathbf{p}_{-k}\right)$ to represent $\mathrm{SU}_{k}$ 's utility function.

Before establishing the uniqueness of NE in the power allocation game, we need the following definition. The best response function of $\mathrm{SU}_{\mathrm{k}}$ is given by

$$
u_{k}\left(\mathbf{p}_{-k}\right)=\left\{p_{k} \in \mathbf{p}_{k}: u_{k}\left(p_{k}, \mathbf{p}_{-k}\right) \geq u_{k}\left(p_{k}^{\dagger}, \mathbf{p}_{-k}\right), p_{k}^{\dagger} \in \mathbf{p}_{k}\right\} .
$$

The goal in CR MIMO-BCs is to achieve the optimal power allocation $p_{k}$. From (22), we can obtain $u_{k}^{\prime}\left(p_{k}^{\dagger}, \mathbf{p}_{-k}\right)=0$. We assume that $\mathrm{SU}_{k}$ has target $\operatorname{SINR} \gamma_{k}$ corresponding to the best response. The target SINR must be admissible and satisfies [33]

$$
\sum_{k=1}^{K} \frac{\gamma_{k}}{1+\gamma_{k}}<M
$$

In this case, the corresponding utility function is

$$
u_{k}\left(\gamma_{k}\right)=\frac{\gamma_{k}}{\gamma_{k}+\alpha}
$$

where $\alpha(\alpha \geq 1)$ is a constant for all SUs' channel and is defined as adjustable parameter. Given the existence of the NE solution, we need to propose an algorithm for SUs to reach NE. In CR MIMO-BCs, the NE is not Pareto optimal because each SU independently selects strategies thus affects each other. Each SU must pay off some price in selecting the strategy. A linear pricing function is commonly used because of its implementation simplicity, which allows a distributed implementation where the price can be broadcast by the base station to all terminals $[30,34]$. In this paper, we use independent linear pricing function $\lambda p_{k}$, where $\lambda$ is a constant, called pricing factor. The net utility function is $u_{k}=\gamma_{k} /\left(\gamma_{k}+\alpha\right)-\lambda p_{k}$. The optimal power allocation problem for $\mathrm{SU}_{k}$ is represented as

$$
\arg \max u_{k}=\arg \max \left(\frac{\gamma_{k}}{\gamma_{k}+\alpha}-\lambda p_{k}\right) .
$$

Taking the derivative of $u_{k}$ in (25), we have

$$
\frac{\partial u_{k}}{\partial p_{k}}=\frac{\partial u_{k}}{\partial \gamma_{k}} \cdot \frac{\partial \gamma_{k}}{\partial p_{k}}-\lambda=\frac{\rho_{k} \alpha}{\left(\gamma_{k}+\alpha\right)^{2}}-\lambda=0 .
$$

Solving (26), we can obtain optimal power allocation to $\mathrm{SU}_{\mathrm{k}}$ expression as

$$
p_{k}=\sqrt{\frac{\alpha}{\lambda \rho_{k}}}-\frac{\alpha}{\rho_{k}}, \rho_{k}=\frac{\mathbf{w}_{k}^{H} \mathbf{R}_{k} \mathbf{w}_{k}}{\sum_{i \neq k} p_{i} \mathbf{w}_{i}^{H} \mathbf{R}_{k} \mathbf{w}_{i}+\sigma_{k}^{2}} .
$$

From (27), power allocation is a process of dynamic interaction. Now, the problem is whether the $p_{k}$ defined above exists and whether it is globally optimal (when it exists) [35]. We need to find a NE that allows the system to achieve the optimum performance. According to the Nash theorem [36], the following two conditions must be satisfied. First, power allocation $p_{k}$ is not empty, closed, and bounded convex set in the Euclidean space. Second, the net utility function is a continuous function and is quasiconcave in $p_{k}$. The first condition is satisfied as each SU's selection action set is defined as $\left[0, \widehat{p}_{k}\right]$. For the second condition, the second derivative of the net utility function is given by

$$
\frac{\partial^{2} u_{k}}{\partial p_{k}^{2}}=\frac{\partial\left(\frac{\rho_{k} \alpha}{\left(\gamma_{k}+\alpha\right)^{2}}-\lambda\right)}{\partial p_{k}}=\frac{-2 \rho_{k}^{2} \alpha}{\left(\rho_{k} p_{k}+\alpha\right)^{3}}<0 .
$$

From (28), the net utility function is a continuous function and is concave in $p_{k}$. The concave function is also a quasi-concave function. Hence, the corresponding power allocation solution must exist.

If the best response function of SUs is a standard function, then NE in this game will be unique. A function $u(\mathbf{p})$ is said to be a standard function if it satisfies the following three properties [37]:

1. Positivity: $u(\mathbf{p})>0$.

2. Monotonicity: If $\mathbf{p} \geq \mathbf{p}^{\dagger}$, then $u\left(\mathbf{p}^{\dagger}\right) \geq u(\mathbf{p})$.

3. Scalability: For all $\mu>1, \mu u\left(\mathbf{p}^{\dagger}\right) \geq u(\mu \mathbf{p})$.

To show that the best response function of $\mathrm{SU}_{k}$ is a standard function, we prove the above three properties as follows:

1. Positivity: Since each SU can obtain a certain utility through power allocation and satisfies $u\left(p_{k}\right)>0$.

2. Monotonicity: Since $u\left(p_{k}\right)>0$, if $p_{k} \leq p_{k}^{\dagger}$, then $\rho_{k} \geq \rho_{k}^{\dagger}$. We can derive $\frac{\gamma_{k}}{\gamma_{k}+\alpha}-\lambda p_{k} \geq 0 \Rightarrow \frac{\rho_{k} p_{k}}{\rho_{k} p_{k}+\alpha} \geq$ 


$$
\begin{aligned}
& \lambda p_{k} \Rightarrow \frac{\alpha}{\lambda} \geq \frac{\alpha^{2}}{\rho_{k}}, u\left(p_{k}\right)-u\left(p_{k}^{\dagger}\right)=\left(\sqrt{\frac{\alpha}{\lambda \rho_{k}}}-\frac{\alpha}{\rho_{k}}\right)- \\
& \left(\sqrt{\frac{\alpha}{\lambda \rho_{k}^{\dagger}}}-\frac{\alpha}{\rho_{k}^{\dagger}}\right) \geq \frac{\left(\alpha \rho_{k}^{\dagger}-\alpha \sqrt{\rho_{k} \rho_{k}^{\dagger}}-\alpha \rho_{k}^{\dagger}+\alpha \rho_{k}\right)}{\rho_{k} \rho_{k}^{\dagger}}=\frac{\left(\rho_{k}-\rho_{k} \rho_{k}^{\dagger}\right) \alpha}{\rho_{k} \rho_{k}^{\dagger}} \geq 0
\end{aligned}
$$

3. Scalability: For $\mu>1, \rho_{\mu}=\frac{\mathbf{w}_{k}^{H} \mathbf{R}_{k} \mathbf{w}_{k}}{\sum_{i \neq k} \mu p_{i} \mathbf{w}_{i}^{H} \mathbf{R}_{k} \mathbf{w}_{i}+\sigma_{k}^{2}}$, then $\rho_{\mu}<\rho_{k}, \mu u\left(p_{k}\right)-u\left(\mu p_{k}\right)=\mu\left(\sqrt{\frac{\alpha}{\lambda \rho_{k}}}-\frac{\alpha}{\rho_{k}}\right)^{k}-$ $\left(\sqrt{\frac{\alpha}{\lambda \rho_{\mu}}}-\frac{\alpha}{\rho_{\mu}}\right) \geq \sqrt{\frac{\alpha}{\rho}}\left(\frac{\mu}{\sqrt{\rho_{k}}}-\frac{1}{\sqrt{\rho_{\mu}}}\right)+\frac{\alpha}{\rho_{\mu}}-\frac{\mu \alpha}{\rho_{k}}=$ $\alpha\left(\frac{\rho_{k}-\sqrt{\rho_{k} \rho_{\mu}}}{\rho_{k} \rho_{\mu}}\right)>0$

From the propositions in the previous section, the best response of SUs is to maximize its individual net utility function.

\section{Iterative algorithm}

In order to better understand the analytical structure of SINR balancing, an iterative algorithm is proposed to obtain the optimal beamforming matrix and power allocation, where each iteration has two steps. In the first step, the beamforming vectors $\mathbf{w}_{k}$ is fixed, and the optimal power vector $\mathbf{p}$ is obtained. In the second step, we fix the update power vector $\mathbf{p}$ to get the corresponding optimal beamforming matrix $\mathbf{w}_{k}$. One key property of the iterative algorithm is that, for a given beamforming matrix, the optimal power allocation vector $\mathbf{p}$ should satisfy the following two necessary conditions

$$
\begin{aligned}
& \frac{\operatorname{SINR}_{k}\left(\mathbf{w}_{k}, \mathbf{p}\right)}{\gamma_{k}}=l_{1}\left(\mathbf{w}, p_{\text {th }}^{(1)}\right) \\
& \sum_{k} g_{1, k} p_{k} \mathbf{w}_{k}^{H} \mathbf{w}_{k}=p_{\text {th }}^{(1)}
\end{aligned}
$$

where $l_{1}\left(\mathbf{w}, p_{\text {th }}^{(1)}\right)$ is called SU's SINR level for the given beamforming matrix. Here, we assume that there is only one PU that it is enough to demonstrate the key aspects of spectrum sharing while avoiding unnecessary complications. The optimal power allocation leads to the balanced SINR for all SUs, and it satisfies the interference constraints in (8) with equality. According to the definition of $\operatorname{SINR}_{k}\left(\mathbf{w}_{k}, \mathbf{p}\right)$ in (5), $l_{1}\left(\mathbf{w}, p_{\text {th }}^{(1)}\right)$ can be represented as [38]

$$
\mathbf{C} \tilde{\mathbf{p}}=l_{1}\left(\mathbf{w}, p_{\text {th }}^{(1)}\right) \mathbf{B} \tilde{\mathbf{p}}
$$

where $\mathbf{C}=\left[\begin{array}{cc}\mathbf{I}_{K \times K} & 0_{K \times 1} \\ \mathbf{g}_{1}^{T} & -p_{\text {th }}^{(1)}\end{array}\right], \mathbf{B}=\left[\begin{array}{cc}\tilde{\mathbf{R}} & \Psi \\ \mathbf{0}_{1 \times K} & 0\end{array}\right]$, and $\tilde{\mathbf{p}}=$ $\left[\mathbf{p}^{T}, 1\right]^{T}$, i.e., $\tilde{\mathbf{p}}$ is a $(K+1) \times 1$ vector with $[\tilde{\mathbf{p}}]_{K+1}=1$, where $\mathbf{g}_{1}=\left[g_{1,1}, \cdots, g_{1, K}\right]^{T}$. Let $\tilde{\mathbf{R}}$ and $\Psi$ be respectively defined as

$$
\begin{aligned}
& \tilde{\mathbf{R}}=\left\{\begin{array}{cc}
\mathbf{w}_{k}^{H} \mathbf{R}_{k} \mathbf{w}_{k} / \mathbf{w}_{i}^{H} \mathbf{R}_{k} \mathbf{w}_{i} & k \neq i \\
0 & k=i
\end{array}\right. \\
& \Psi=\left[\sigma_{1}^{2} / \mathbf{w}_{k}^{H} \mathbf{R}_{1} \mathbf{w}_{k}, \cdots, \sigma_{k}^{2} / \mathbf{w}_{k}^{H} \mathbf{R}_{k} \mathbf{w}_{k}\right]
\end{aligned}
$$

In (31), $\mathbf{C}$ is nonsingular, and we have

$$
\begin{aligned}
& \Phi_{0}\left(\mathbf{w}_{k}, p_{\text {th }}^{(1)}\right) \tilde{\mathbf{p}}=\frac{1}{l_{1}\left(\mathbf{w}, p_{\text {th }}^{(1)}\right)} \tilde{\mathbf{p}} \\
& \Phi_{0}\left(\mathbf{w}_{k}, p_{\text {th }}^{(1)}\right)=\mathbf{C}^{-\mathbf{1}} \mathbf{B}=\left[\begin{array}{cc}
\tilde{\mathbf{R}} & \Psi \\
\frac{\mathbf{g}_{1}^{T} \tilde{\mathbf{R}}}{p_{\text {th }}^{(1)}} & \frac{\mathbf{g}_{1}^{T} \Psi}{p_{\text {th }}^{(1)}}
\end{array}\right] .
\end{aligned}
$$

For a given beamforming vector $\mathbf{w}_{k}, l_{1}\left(\mathbf{w}, p_{\text {th }}^{(1)}\right)$ equals the reciprocal of the largest eigenvalue of non-negative matrix $\Phi_{0}\left(\mathbf{w}_{k}, p_{\text {th }}^{(1)}\right)[38,39]$ and is given by

$$
l_{1}\left(\mathbf{w}, p_{\mathrm{th}}^{(1)}\right)=\frac{1}{\max \lambda\left(\Phi_{0}\left(\mathbf{w}_{k}, p_{\mathrm{th}}^{(1)}\right)\right)}
$$

For fixed beamformers, the transmit powers can be adjusted so as to guarantee the target SINR values with minimal total power. However, an update of the power allocation makes it necessary to reconsider the optimality of the beamformers. Therefore, to reach the NE of the joint beamforming subgame and power control subgame, we combine (5), (20) and (36) to obtain the utility function as follows

$$
\begin{aligned}
J_{k}\left(p_{k}, \mathbf{w}_{k}, \mathbf{w}_{-k}\right)= & \tau_{k}\left(\gamma_{k}-p_{k} l_{1}\left(\tilde{\mathbf{w}}_{k}, \tilde{\mathbf{w}}_{-k}\right)\right) \\
& +\ln \mathbf{w}_{k}^{H} \mathbf{h}_{k}^{H} \mathbf{R}_{k}\left(\tilde{\mathbf{w}}_{-k}\right)^{-1} \mathbf{h}_{k} \mathbf{w}_{k} \\
& -\ln \mathbf{w}_{k}^{H} \mathbf{R}_{k}^{*}\left(\tilde{\mathbf{w}}_{-k}\right)^{-1} \mathbf{w}_{k}
\end{aligned}
$$

where the utility function is subject to the target SINR constraints, corresponds to an optimal ensemble of SUs' beamformers and power allocations. The corresponding iterative game algorithm is given in Algorithm 1.

\section{Numerical simulation results and discussions}

In this section, numerical results are provided to examine the performance of our proposed algorithms with finite numbers of secondary users and antennas. For Rayleigh fading, the power gain matrix $\mathbf{g}_{1}$ can be described as exponentially distributed and assume $g_{1,1}, g_{1,2}, \cdots g_{1, K}$ are unit-mean and mutually independent [35]. The elements of the channel matrix $\mathbf{R}_{k}$ are assumed to be circularly symmetric complex Gaussian with zero mean and unity variance. The noise covariance matrix is assumed to be the identity matrix. We consider CBS with five antennas $(M=5)$, the four SUs each equipped with single antenna. In addition, an interference threshold of 0.01 is used to protect PU, i.e., $p_{\mathrm{th}}^{(1)}=-20 \mathrm{~dB}$. The SUs power allocation and the target SINRs are initialized $\mathbf{p}=\mathbf{0 . 0 1}_{4}$ and $\gamma=$ $\{2,1.9,1.8,1.7\}$, respectively, where we set target SINRs for SUs that they satisfy the admissibility condition in (23). The total transmission power $p$ and power allocations to SUs are defined in $\mathrm{dB}$ relative to the noise power. For each scenario, 10,000 simulation runs are used for average. The following parameters are used in all numerical 


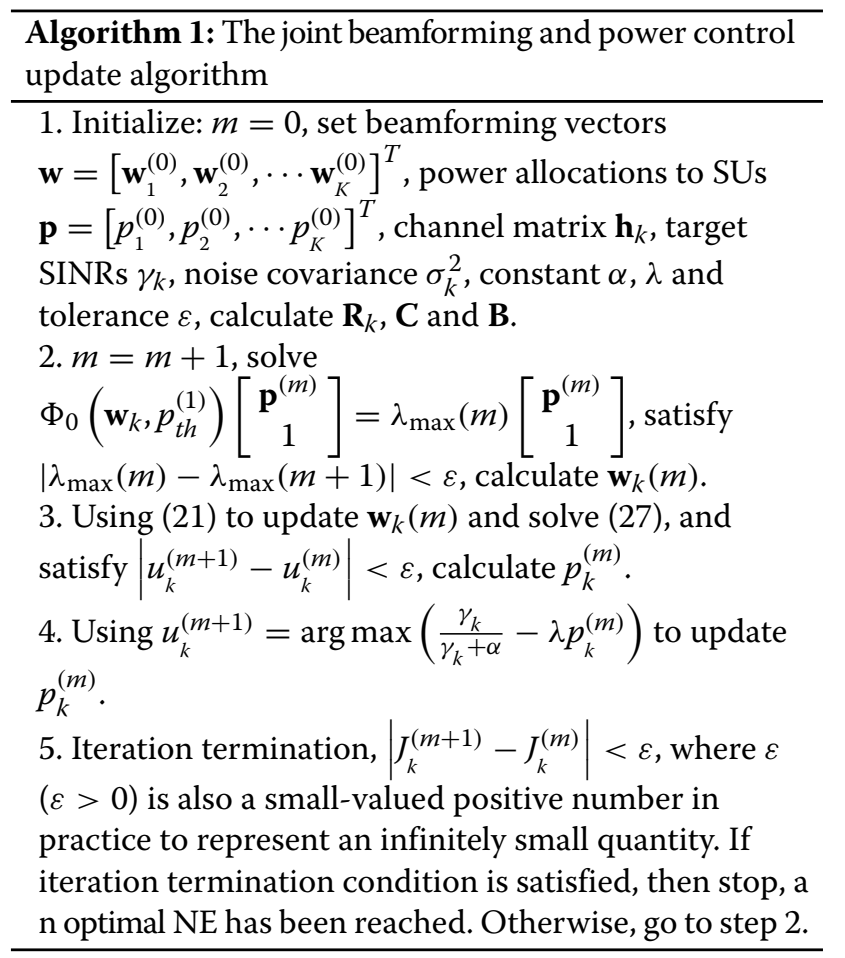

simulations unless stated otherwise: $\alpha=1, \lambda=0.1$, and $\varepsilon$ are small-valued positive numbers and equal to 0.005 , respectively.

Figure 4 shows the normalized maximum SINR and the total transmission power $p$ for the proposed algorithm with different iteration steps $m$. It is assumed that CBS is composed of five omnidirectional uniform linear arrays. The linear arrays are separated by a distance $10 \lambda_{0}$, where $\lambda_{0}$ is the signal wavelength. From Fig. 4 , we can observe that the proposed algorithm converges very fast $(m \geq 30)$

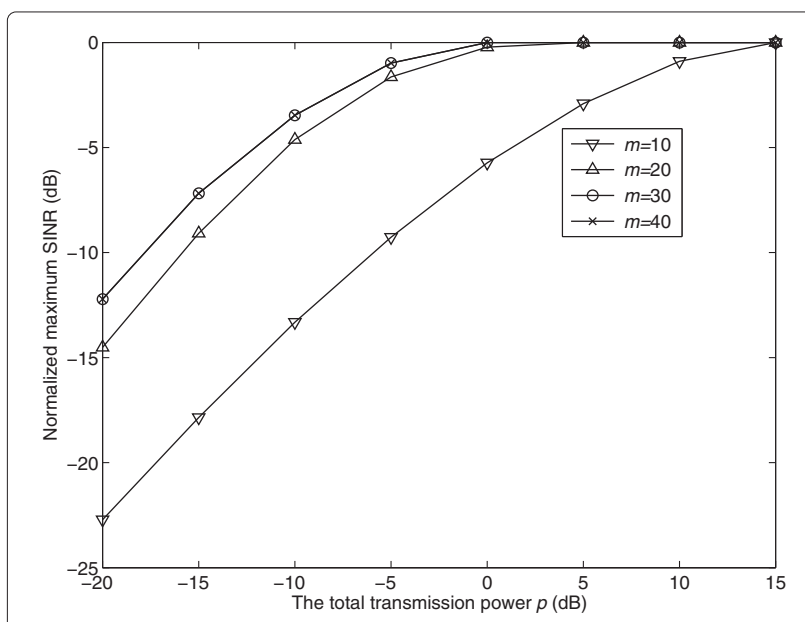

Fig. 4 Normalized maximum SINR and the total transmission power $p$ curves for the proposed algorithm with different iteration steps $m$



Fig. 5 Normalized beam pattern power for the proposed algorithm

to the normalized maximization SINR. As expected, the total transmission power $p$ can impact the performance very significantly.

Figure 5 illustrates the normalized beampattern power for the proposed algorithm. SUs are respectively located in the directions of $\phi_{1}=-102^{\circ}, \phi_{2}=-26^{\circ}, \phi_{3}=44^{\circ}$, and $\phi_{4}=112^{\circ}$ relative to CBS, where $\phi$ is the azimuth angle. It is assumed that the change in direction of arrival (DOA) of input waves to the SUs may be up to $\pm 3^{\circ}$ arbitrarily. One PU is located at the direction $\phi=60^{\circ}$. It can be seen that each beampattern has minimum interference at the directions of the mainlobes of the other beampatterns, but they do not affect beamforming directions at SU.

Figures 6 and 7 show the variations of SUs power and SINRs for variable target SINRs. These features are useful in CR MIMO-BCs, where different QoS requirements

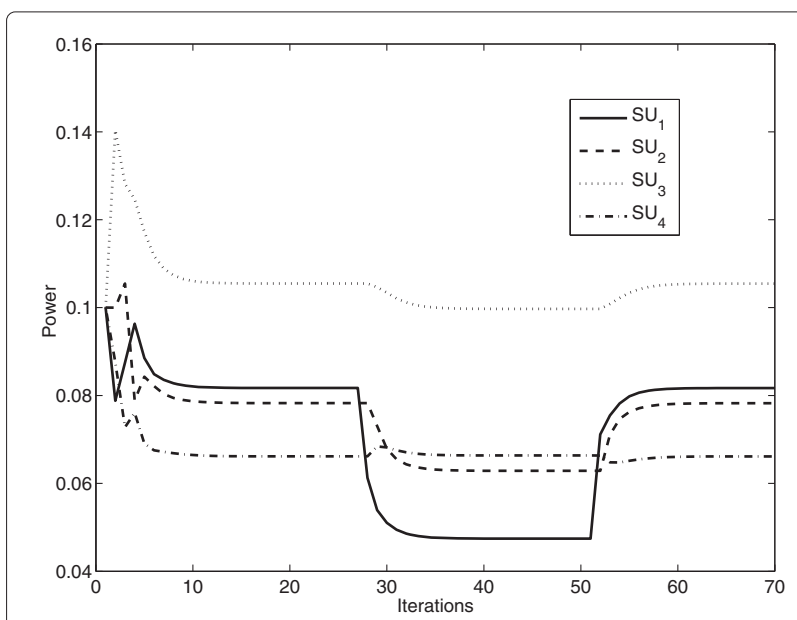

Fig. 6 SU's power versus iterations for variable target SINRs 


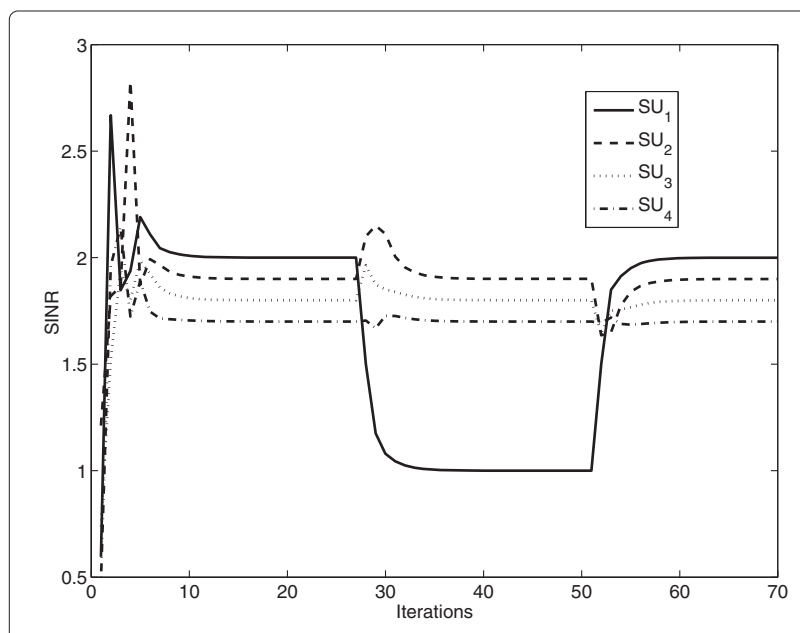

Fig. 7 SU's SINR versus iterations for variable target SINRs

may lead to the target SINRs variation, which makes a NE configuration be achieved. We then vary the first SU's target SINR to a new value $\gamma_{1}^{*}=1$, leading the algorithm to reach a new NE configuration. Finally, we change SINR back to its old value $\gamma_{1}^{*}=2$, restoring essentially the same $\mathrm{NE}$ configuration, and it achieves a faster convergence speed.

Figure 8 plots the total transmission power $p$ versus the numbers of iterations under different values of $\lambda$ and $\alpha$ for the proposed algorithm. It can be seen that the number of iterations decreases as the values of the pricing factor $\lambda$ increases. This is because SUs will pay off higher pricing with the increase of the pricing factor. In addition, setting different values of $\lambda$ and $\alpha$, we can find a trade-off between the system performance and convergence speed for the proposed algorithm. As a result, we can conclude that the



Fig. 8 The total transmission power $p$ versus the numbers of iterations under different values of $\lambda$ and $\alpha$ for the proposed algorithm proposed bound is a useful tool for finding the optimal value of $\lambda$ and $\alpha$.

\section{Conclusions}

We have formulated the SINR balancing designs of CR MIMO-BCs into optimization problem. Exploiting BCMAC duality, we have formed joint transmit beamforming and power allocation as noncooperative separable game. We have then exploited the convex theory of noncooperative game to solve the optimalization problem. Finally, we have proposed an iterative algorithm to reach the NE of the joint beamforming subgame and power control subgame. Numerical results are provided to validate the optimality and the convergence of the proposed algorithm. Moreover, a trade-off between the system performance and convergence speed for the proposed algorithm can be flexibly controlled by setting different parameter values.

\section{Competing interests}

The authors declare that they have no competing interests.

\section{Acknowledgements}

The authors acknowledge the support from the National Natural Science Foundation of China (Grant No:: 61472094; 61261018), Guangxi Natural Science Foundation under Grants 2014GXNSFGA118007, and the open research fund of the National Mobile Communications Research Laboratory, Southeast University (No. 2015D05).

\section{Author details}

${ }^{1}$ Library of Guilin University of Electronic Technology, Jinji Road, 541004 Guilin China. ${ }^{2}$ Guangxi Key Laboratory of Wireless Wideband Communication and Signal Processing, Jinji Road, 541004 Guilin, China. ${ }^{3}$ National Mobile Communications Research Laboratory, Southeast University, 210096 Najing, China.

Received: 4 January 2016 Accepted: 27 March 2016

Published online: 12 April 2016

\section{References}

1. C Sun, YD Alemseged, HN Tran, H Harada, Transmit power control for cognitive radio over a Rayleigh fading channel. IEEE Trans. Veh. Tech. 59(4), 1847-1857 (2010)

2. S Stotas, A Nallanathan, On the throughput and spectrum sensing enhancement of opportunistic spectrum access cognitive radio networks. IEEE Trans. Wireless Commun. 1(1), 97-107 (2012)

3. M Hasegawa, H Hirai, K Nagano, H Harada, K Aihara, Optimization for centralized and decentralized cognitive radio networks. Proc. IEEE. 102(4), 574-584 (2014)

4. S Akin, MC Gursoy, Performance analysis of cognitive radio systems with imperfect channel sensing and estimation. IEEE Trans. Commun. 63(5), 1554-1566 (2015)

5. SJ Kim, G Li, GB Giannakis, Multi-band cognitive radio spectrum sensing for quality-of-service traffic. IEEE Trans. Wireless Commun. 10(10), 3506-3515 (2011)

6. W Yin, P Ren, Q Du, Y Wang, Delay and throughput oriented continuous spectrum sensing schemes in cognitive radio networks. IEEE Trans. Wireless Commun. 11(6), 2148-2159 (2012)

7. $V$ Asghari, S Aissa, Impact of detection uncertainties on the performance of a spectrum-sharing cognitive radio with soft sensing. IEEE Trans. Veh. Tech. 61(7), 3272-3276 (2012)

8. Y Huang, G Zheng, M Bengtsson, KK Wong, L Yang, B Ottersten, Distributed multicell beamforming design approaching pareto boundary with max-min Fairness. IEEE Trans. Wireless Commun. 11(8), 2921-2933 (2012) 
9. Y Rahulamathavan, K Cumanan, S Lambotharan, A mixed SINR-balancing and SINR-target-constraints-based beamformer design technique for spectrum-sharing networks. IEEE Trans. Veh. Tech. 60(9), 4403-4414 (2011)

10. HXiao, S Ouyang, CX Wang, Cognitive radio MIMO Gaussian broadcast channels with the power constraint. Wireless Personal Commun. 68(3), 769-778 (2013)

11. DN Nguyen, M Krunz, Power minimization in MIMO cognitive networks using beamforming games. IEEE J. Sel. Areas in Commun. 31(5), 916-925 (2013)

12. R Zhang, F Gao, YC Liang, Cognitive beamforming made practical: effective interference channel and learning-throughput tradeoff. IEEE Trans. Commun. 58(2), 706-718 (2010)

13. EA Gharavol, YC Liang, K Mouthaan, Robust downlink beamforming in multiuser MISO cognitive radio networks with imperfect channel-state information. IEEE Trans. Veh. Tech. 59(6), 2852-2860 (2010)

14. H Du, T Ratnarajah, M Pesavento, Joint transceiver beamforming in MIMO cognitive radio network via second-order cone programming. IEEE Trans. Signal Process. 60(2), 781-792 (2012)

15. DN Nguyen, M Krunz, Price-based joint beamforming and spectrum management in multi-antenna cognitive radio networks. IEEE J. Sel. Areas Commun. 30(11), 2295-2305 (2012)

16. S Choi, H Park, T Hwang, Optimal beamforming and power allocation for sensing-based spectrum sharing in cognitive radio networks. IEEE Trans. Veh. Tech. 63(1), 412-417 (2014)

17. IW Lai, L Zheng, CH Lee, CW Tan, Beamforming duality and algorithms for weighted sum rate maximization in cognitive radio networks. IEEE J. Sel. Areas Commun. 33(5), 832-847 (2015)

18. M Schubert, $\mathrm{H}$ Boche, Solution of the multiuser downlink beamforming problem with individual SINR constraints. IEEE Trans. Veh. Tech. 53(1), 18-28 (2004)

19. C Lacatus, D C Popescu, Adaptive interference avoidance for dynamic wireless systems: a game-theoretic approach. IEEE J. Sel. Topics Signal Process. 1(1), 189-202 (2007)

20. L Zhang, Y Xin, YC Liang, Weighted sum rate optimization for cognitive radio MIMO broadcast channels. IEEE Trans. Wireless Commun. 8(6), 2950-2959 (2009)

21. Y Huang, S He, S Jin, W Chen, Decentralized energy efficient coordinated beamforming for multicell systems. IEEE Trans. Veh. Tech. 63(9), 4302-4314 (2014)

22. YY He, S Dey, Sum rate maximization for cognitive MISO broadcast channels: beamforming design and large systems analysis. IEEE Trans. Wireless Commun. 13(5), 2383-2401 (2014)

23. L Zhang, R Zhang, YC Liang, Y Xin, HV Poor, On Gaussian MIMO BC-MAC duality with multiple transmit covariance constraints. IEEE Trans. Infor. Theory. 58(4), 2064-2078 (2012)

24. G Dartmann, X Gong, W Afzal, G Ascheid, On the duality of the max-min beamforming problem with per-antenna and per-antenna-array power constraints. IEEE Trans. Wireless Commun. 58(4), 2064-2078 (2012)

25. HT Do, SY Chung, Linear beamforming and superposition coding with common information for the gaussian MIMO broadcast channel. IEEE Trans. Commun. 57(8), 2484-2494 (2009)

26. W Yu, Uplink-downlink duality via minimax duality. IEEE Trans. Infor. Theory. 52(2), 361-374 (2006)

27. H Park, SH Park, JS Kim, I Lee, SINR balancing techniques in coordinated multi-cell downlink systems. IEEE Trans. Wireless Commun. 12(2), 626-635 (2013)

28. S Vishwanath, N Jindal, A Goldsmith, Duality, achievable rates, and sum-rate capacity of Gaussian MIMO broadcast channels. IEEE Trans. Infor. Theory. 49(10), 2658-2668 (2003)

29. L Zhang, YC Liang, Y Xin, Joint beamforming and power allocation for multiple access channels in cognitive radio networks. IEEE J. Sel. Areas in Commun. 26(1), 38-51 (2008)

30. Y Yu, T Zhang, Danny HK Tsang, Joint pricing and power allocation for dynamic spectrum access networks with Stackelberg game model. IEEE Trans. Wireless Commun. 10(1), 12-19 (2011)

31. RA lltis, S kim, D A Hong, Noncooperative iterative MMSE beamforming algorithms for ad hoc networks. IEEE Trans. Commun. 54(4), 748-759 (2006)

32. L Zhang, W Liu, L Yu, Performance analysis for finite sample MVDR beamformer with forward backward processing. IEEE Trans. Signal Process. 59(5), 2427-2431 (2011)
33. DC Popescu, DB Rawat, O Popescu, M Saquib, Game-theoretic approach to joint transmitter adaptation and power control in wireless systems. IEEE Trans. Syst. Man Cybern. 40(3), 675-682 (2010)

34. SH Moon, SR Lee, JS Kim, I Lee, Channel quantization for block diagonalization with limited feedback in multiuser MIMO downlink channels. J. Commun. Netw. 16(1), 1-9 (2014)

35. G Scutari, DP Palomar, MIMO cognitive radio: a game theoretical approach. IEEE Trans. Signal Process. 58(2), 761-780 (2010)

36. M Felegyhazi, JP Ubaux, L Buttyan, Nash equilibria of packet forwarding strategies in wireless ad hoc networks. IEEE Trans. Mobile Comput. 5(5), 463-476 (2006)

37. M Rasti, AR Sharafat, B Seyfe, Pareto efficient and goal driven power control in wireless networks: a game-theoretic approach with a novel pricing scheme. IEEE/ACM Trans. Netw. 17(2), 556-569 (2009)

38. W Yang, G Xu, in The Optimal Power Assignment for Smart Antenna Downlink Weighting Vector Design. IEEE Proceedings of 48th VTC (48th IEEE Vehicular Technology Conference, Ottawa, Canada, 1998), pp. 485-488

39. G Zheng, KK Wong, B Ottersten, Robust cognitive beamforming with bounded channel uncertainties. IEEE Trans. Signal Process. 57(12), 4871-4881 (2009)

\section{Submit your manuscript to a SpringerOpen ${ }^{\circ}$ journal and benefit from:}

- Convenient online submission

- Rigorous peer review

- Immediate publication on acceptance

- Open access: articles freely available online

- High visibility within the field

- Retaining the copyright to your article

Submit your next manuscript at $>$ springeropen.com 